\title{
Surface-imprinted biosensors for the detection of proteins, cancer markers and viruses
}

\author{
Yantian Wang ${ }^{1,3,4, * \$}$, Yingjie $\mathbf{Y u}^{1,3}$, Jonathan Sokolov ${ }^{1}$, Kalle Levon ${ }^{2}$ and Miriam Rafailovich ${ }^{1, *, \#}$ \\ ${ }^{1}$ Department of Materials Science and Chemical Engineering, Stony Brook University, Stony Brook, \\ NY 11794-2275; ${ }^{2}$ Department of Chemical and Biomolecular Engineering, \\ New York University Tandon School of Engineering, Brooklyn, NY 11201, USA.
}

\begin{abstract}
Biosensors using artificial recognition elements have generated intensive interest from scientists and medical professionals in recent years. Their high stability and sensitivity make them easier and less costly to use, store, and manufacture than sensors based on biological recognition elements. Surfaceimprinted sensors using the organic self-assembled monolayers (SAMs) of functionalized oligomers on gold surfaces have the advantages of high contact areas, fast response, easy construction, as well as integration of the recognition element with the transducer, all of which can lead to high sensitivity. The possibility of using SAM molecules with different end groups offers the flexibility of changing the affinity of the sensor to the target biomolecules while modification of the gold surface roughness enables imprinting bio-macromolecules much larger than the length of the SAM molecules. Co-dissolution of the bio-macromolecules and the organic molecules in a blend of aqueous/organic solvents ensures the formation of SAMs and at the same time maintains the viability and configuration of the biomacromolecules. Hence the synergy established between the substrate topography, the surface chemistry, the imprinted SAM, and the molecular structure of the analyte is the essential element
\end{abstract}

\footnotetext{
*Corresponding author

\$yttwang@gmail.com

"Miriam.Rafailovich@stonybrook.edu

${ }^{3}$ Co-first author

${ }^{4}$ Present address: Amway, 7575 Fulton Street Ada, MI 49355, USA.
}

for the successful construction of the biosensor. This review article focuses on the recent achievements in the development of the surface-imprinted biosensors for proteins, cancer markers and viruses, from multiple groups working in this area.

KEYWORDS: biosensor, surface molecular imprinting, self-assembled monolayer.

\section{Introduction}

A biosensor includes two key components: a bioreceptor (recognition element) and a transducer. The recognition element is responsible for selective binding of the target analytes, and the transducer produces the signal in accordance with the binding amount of the target analyte. The signal transition principle of the transducer can be based on the mass [1], optical [2, 3], sound [4-6], fluorescence [7-9], electrochemical [10-23], or thermal response [24]. The recognition elements are usually biological agents which can be enzymes [25-27], antibodies [28-30], DNA [26, 28, 31, 32], microbes [33-41], plant or animal cells [25, 42, 43], etc. While these biological agents give excellent sensitivity and selectivity to the recognition elements, they also face limitations in the practical applications, owing to their instability against harsh environments such as high temperatures, extreme $\mathrm{pH}$ and organic solvents [44-47]. Also, the synthesis of the antibodies can be expensive and time consuming $[46,48]$. To alleviate or overcome these problems, various artificial recognition elements have been developed [49, 50], among which molecular-imprinted (MI) recognition elements have attracted intensive interest for research 
and investigation, and have brought dramatic increase in their application in new biosensors for the past two decades [51]. The principle of molecular imprinting is analogues to the formation of the antibodies proposed by Pauling [52, 53], in which a cross-linked polymer is formed around a template molecule. After removal of the template, an imprint "foot-print" containing functional groups capable of chemical interaction remains in the polymer matrix. The shape of the imprint and the arrangement of the functional groups are complementary to the structure of the template. This imprinted polymer matrix will then have a higher affinity to re-absorb and bind to the same template molecules compared to other molecules [54]. The traditional 3D molecular imprinting, although has the advantage of high precision due to the chemical interaction of the binding sites, has a relatively slow response time, as the recognition sites buried inside of the matrix are not subject to immediate access by the target molecules [55, 56]. Also, the bulk polymer recognition element doesn't enable the integration with the transducer. Surface imprinting using the self-assembled monolayers (SAMs), as a subcategory of molecular imprinting method, has overcome the problems of slow response of the traditional MI element, owing to the extremely small thickness of the sensor, which offers the benefit of faster response and the integration of the recognition element with the transducer [57]. Also, the price of the molecularly imprinted recognition element could be two orders of magnitude lower than those made with the antibodies [58]. Application of surface imprinting in the detection of a variety of small molecules has achieved success in the recent decades [57, 59-61]; however, the application of the technique in the detection of bio-macromolecules is relatively new, mainly due to the challenges of maintaining the viability and configuration of the bio-macromolecules during the sensor fabrication process. As the SAMs formed by the organic molecules are mostly only soluble in organic solvents, while bio-macromolecules will lose viability or change configuration in these solvents, the production of the solutions that dissolve the organic molecules while maintaining the configurations of the bio-macromolecules becomes a key issue. In this article, recent progress made by several research groups in the surface-imprinted biosensor is reviewed, which include sensors for globular proteins, cancer markers and viruses [62].

\section{Review of various technical aspects for the formation of self-assembled monolayers and surface molecular imprinting}

Self-assembly is a process of spontaneous selfassociation of the interlocking components to form a well-organized supramolecular structure that represents the thermodynamic equilibrium under a given set of experimental conditions [63]. SAMs are ordered molecular assemblies formed by the adsorption of an active surfactant on a solid surface [64]. The formation of monolayer of molecules on a solid/liquid interface is driven by specific interactions between the functional groups of the self-assembling molecules and the surface. The process is followed by a self-organization of the molecules which is stabilized by an interplay of non-covalent or covalent interactions among the self-assembling molecules [58, 64]. Because the formation of the SAMs depends on the interaction of the head groups of the molecules and the substrate, the tail groups of the self-assembling molecules can render the surfaces a wide variety of physical/chemical properties, such as a controlled hydrophilicity/hydrophobicity $[65,66]$, affinity to proteins [67], electrostatic charge [68], and electrical conductivity [69].

The major categories of SAMs that have been most widely studied include [64]:

1. Monolayers of fatty acid. This is the result of the spontaneous adsorption of long-chain alkanoic acids $\left(\mathrm{C}_{n} \mathrm{H}_{\mathrm{n}+2} \mathrm{COOH}\right)$ on metal oxide surface. The driving force of the interaction is the formation of a surface salt between the carboxylate anion and the surface metal cation.

2. Monolayers of organosilicon derivatives. This type of SAM is formed by alkylchlorosilanes, alkyloxysilanes, alkylaminosilanes on hydroxylated substrates. The driving force of the formation is in situ formation of the polysiloxane that is connected to the substrate silanol groups (-SiOH) via Si-O-Si bond. In order to form the high quality SAMs of alkyltrichlorosilane derivatives, the humidity of the environment and the amount of the water in the solution have to be carefully controlled. Insufficient water will lead to formation of incomplete monolayer, while excess water will result in facile polymerization in solution and polysiloxane deposition on surface. Usually 
fabrication of this kind of SAM will need to be operated in a glovebox with controlled humidity.

3. Organosulfur adsorbates on metal and semiconductor surfaces. The formation of this type of SAM is based on the fact that sulfur and selenium compounds have a strong affinity to transition metal surfaces [64, 70-72]. Although organosulfur compounds co-ordinate strongly to a variety of metals, most investigations have been carried out on SAMs of thiolates on gold surface. The alkanethiol SAMs were prepared by immersing the pre-cleaned gold substrate in dilute thiol solutions, two distinctive adsorption kinetic steps can be observed: a fast step, which takes a few minutes, by the end of which contact angles are close to its limiting value and the thickness about $80-90 \%$ of its maximum; and a slow step, which lasts a few hours, at the end of which the contact angle and thickness reach their final values. The second step is a crystallization process, during which the molecule chains get out of their disordered state and organize into a crystalline state [64]. Studies on the thermal stability of the thiolate SAMs on gold have shown that desorption of thiol molecules occurs at $170-230{ }^{\circ} \mathrm{C}$, while some loss of surface sulfur can happen at $100{ }^{\circ} \mathrm{C}$ [73, 74].

Besides the SAMs categories reviewed above, there are other less widely studied types of selfassembled monolayers or multilayers, such as alkyl monolayers on silicon [75], multilayers of diphospates [76], etc. Since they don't find as wide applications in biosensors as organosilane SAMs or thiolate SAMs, application of category (2) and (3) monolayers in biosensors will be the focus of review in this article.

Owing to the flexibility to render the surface desired properties, SAM is a valuable choice for manufacturing highly selective sensor surfaces $[77,78]$. Moreover, certain types of SAMs formed under appropriate conditions can offer better mechanical, thermal and electrical stability compared to biological recognition element [79]. Murray et al. discovered the formation of monolayers of bonded organosilane reagents on $\mathrm{SnO}_{2}, \mathrm{TiO}_{2}$, and glassy carbon electrodes under anhydrous reaction conditions. Sagiv et al. were able to form oleophobic monomolecular films that contained mixed monolayers of more than one component on polar surfaces from anhydrous organic solutions [80, 81]. On studying the thermal behavior of the monolayers prepared by covalent, ionic, and physical bonds, Sagiv et al. also found that, the covalently bound octadecylsiloxane (ODS) exhibits exceptional thermal stability, that heating to $110{ }^{\circ} \mathrm{C}$ caused only slight disorientation of the chains in ODS, and no sharp melting was detected by Fourier transformed - infrared spectroscopy (FT-IR) [82]. The formation of the mixed monolayers containing both physisorbed and chemisorbed molecules made the removal of the physisorbed components possible [66, 83, 84]. Sagiv proposed that the remaining skeleton monolayers after removal of the physisorbed molecules have holes of molecular dimensions that may be used as specific adsorption sites. To investigate this hypothesis, they formed the mixed monolayers in the presence of surfactant dyes, which were only physically adsorbed on the surface in contrast to the monolayers that were covalently linked. The dye molecules were washed away, leaving holes in the polymerized silane network. This study can be considered as the first successful surface molecular imprinting experiment [83, 84].

Zhou et al. successfully applied the surface molecular imprinting (SMI) in detecting several chemical species [85-87]. In one of their studies, Zhou et al. used SMI to fabricate the sensor for methylphosphonic acid (MPA), the degradation product of sarin, which is a lethal compound that can be used as the biological weapon. In this approach, MPA (template) and octadecyltrichlorisilane (OTS) were co-adsorbed onto indium tin oxide (ITO)-coated glass substrate under anhydrase condition [87]. The OTS then crosslinked and formed octadecylsiloxane (ODS) monolayer which was covalently bound to the polar surface, while the MPA molecules which was physisorbed could be removed by solvent extraction. The recognition study was performed using a potentiometer. The resulted sensor demonstrated a higher complementary steric and functionality adsorption ability to the templated sensor surface than the un-templated control surface (Fig. 1). The sensor also demonstrated appropriate selectivity, as MPA selectively bound to the ODS film compared to ethylphosphonic acid, propylphosphonic acid, and tert-butylphosphonic 


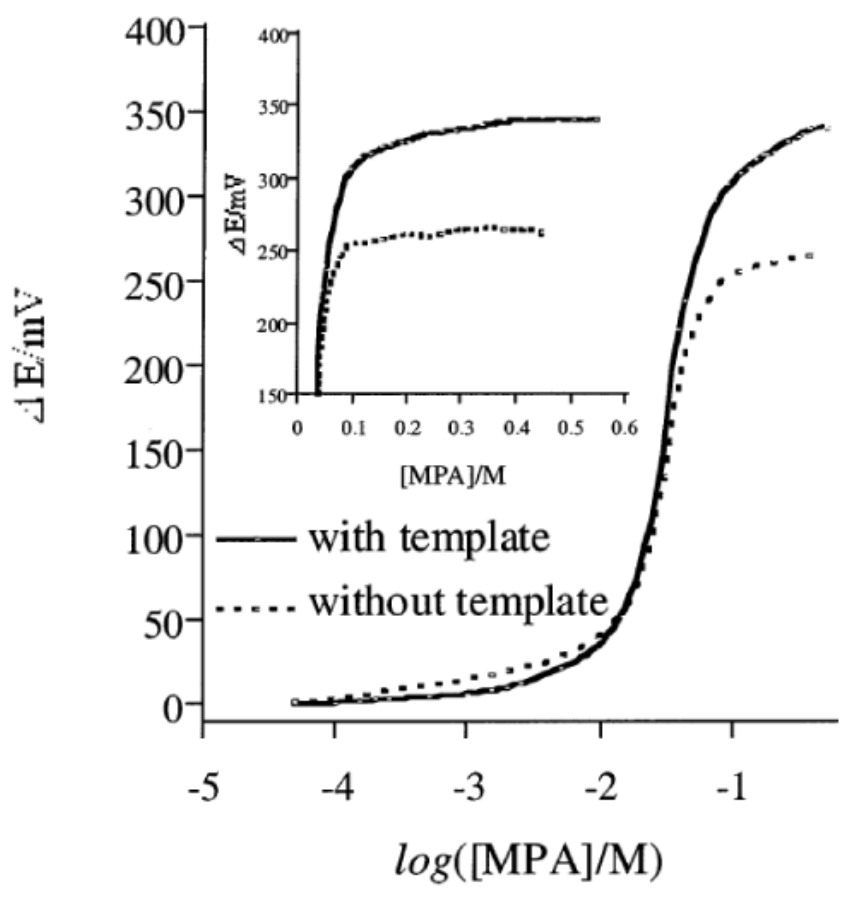

Fig. 1. Potentiometric responses of MPA on the MPA ODS/ITO electrodes with (s) and without template (- - -). [MPA] $\left(\mathrm{CHCl}_{3} / \mathrm{CCl}_{4}\right) 2.5 \times 10^{-2} \mathrm{M}$; [OTS]) $8.0 \times 10^{-4} \mathrm{M}$. Adsorption time $3 \mathrm{~min}$. (Reprinted with permission from Zhou, Y. X., Yu, B., Shiu, E. and Levon, K. 2004, Analytical Chemistry, 76(10), 2689-2693 Copyright (2004) American Chemical Society).

acid; and only at high concentration, a small potential response could be observed for those alkylphosphonic acids (Fig. 2). The response time, evaluated as the time required for a $95 \%$ signal response, was $\sim 50 \mathrm{~s}$ for $1.5 \times 10^{-2} \mathrm{M}$ MPA. The study has shown that high specificity, selectivity, stability, and speed could be realized by the integration of the recognition elements with a nanoscale transducer, here, an ITO-coated electrode [87]. In a separate study, Zhou et al. used similar approach to make the sensor for dipicolinic acid (DPA), a major constituent of bacteria endospores (including B. anthracis spores), and achieved high sensitivity, selectivity, and stability as well as short response time [85].

\section{Detection, quantification, and validation of the technique for sensing analytes of multiple sizes ranging from small biomolecules to proteins, viruses, and bacteria}

Although SMI has been successfully applied in the detection of a variety of small molecules, the investigation of applying SMI in sensing biomolecules are relatively rare and has caught the attention of researchers only in recent years. This is mainly due to the technical difficulties encountered in the fabrication of the sensor. First, the biomolecules are large in size compared to the thickness of the SAMs, which makes the imprinting process less feasible; second, the biomolecules have more complex structures and their conformation are usually sensitive to the environment such as $\mathrm{pH}$, temperature, as well as ionic strength of the solution, all of which lead to a more stringent requirement for the fabrication and the sensing conditions; last and most importantly, the formation of SAMs needs to be carried out in organic solutions, while the biomolecules require aqueous environment and will denature, precipitate or completely change their conformation in the organic solvents. Carefully designed solution is one of the key factors for the successful fabrication of the SMI biosensors. Wang et al. are among the first to explore the possibility of using the SMI in detecting biomacromoleculs including globular proteins, cancer marker proteins, and viruses [15, 10]. In Wang et al.'s approach, 11-Mercapto-1-undecanol was used to form the SAM, which was dissolved in the organic solvent, while bio-macromolecules which were 


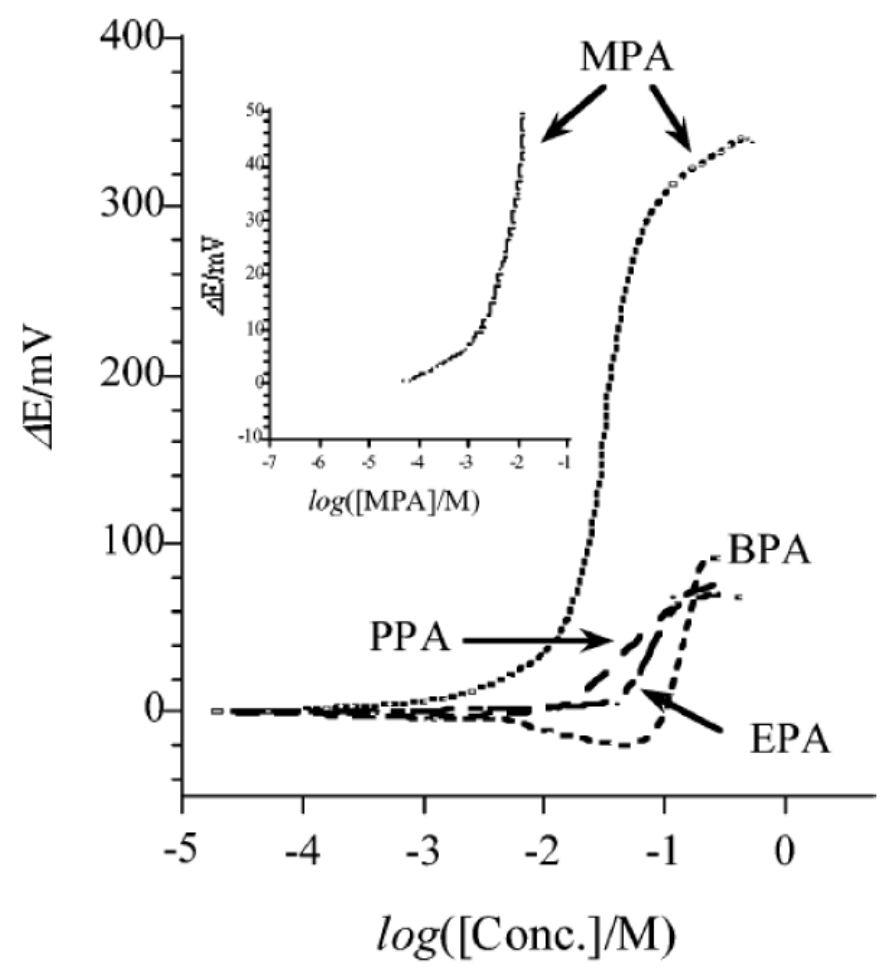

Fig. 2. Potentiometric responses of the MPA cavity sensor for MPA, ethylphosphonic acid (EPA), propylphosphonic acid (PPA), and tert-butylphosphonic acid (BPA). (Reprinted with permission from Zhou, Y. X., Yu, B., Shiu, E. and Levon, K. 2004, Analytical Chemistry, 76(10), 2689-2693 Copyright (2004) American Chemical Society).

used as the template were dissolved in di-ionized water, and the two solutions were then mixed in a $1 / 19$ [organic/aqueous, v/v] ratio. The gold-coated silicon chip was used as the substrate and immersed in the blend solution for $>2$ hours for co-adsorption of the thiol and the template molecules. The extraction of the template biomacromolecules was realized by washing with deionized water. The sensor surface formed by this approach was characterized with atomic force microscopy (AFM), water contact angle measurement, as well as grazing angle-FTIR, which have suggested that the thiol molecules fully covered the gold surface, and had formed the well-organized crystalline SAM. The imprinted sensor chip acted as the integration of recognition element and the transducer, the reaction of which to the target molecules could be tested by connecting to a potentiometer. Both the sensor chip and an $\mathrm{Ag} / \mathrm{AgCl}$ reference electrode were immersed in the test solution, and the potentiometric response of the sensor electrode was measured against the reference electrode as the concentration of the target molecules increased in the solution. Wang et al. has used this technique to build the sensors for hemoglobin, myoglobin, cancer biomarker carcinoembryonic antigen (CEA), and poliovirus, all of which have proven to have desired sensitivity and selectivity. Preliminary quantification study of the CEA sensor has shown a fair match with the result of the enzyme-linked immunosorbent assay (ELISA) test. The smallest detection amount for CEA can be as low as $2.5 \mathrm{ng} / \mathrm{mL}$ (Fig. 3). Wang et al. have proposed the detecting mechanism as a result of the specific hydrophobic/hydrophilic interactions between the surface amino acids of the proteins and the thiol end groups or the gold substrate (Fig. 4). Proteins, cancer markers, as well as the poliovirus capsid have both hydrophobic and hydrophilic domains/regions in their outer surfaces $[88,89]$. It is known that the hydroxyl-terminated thiol acts as a resistant layer to protein adsorption, while gold has much higher affinity to adsorb proteins [90-92]. As a result, the hydroxyl-terminated 

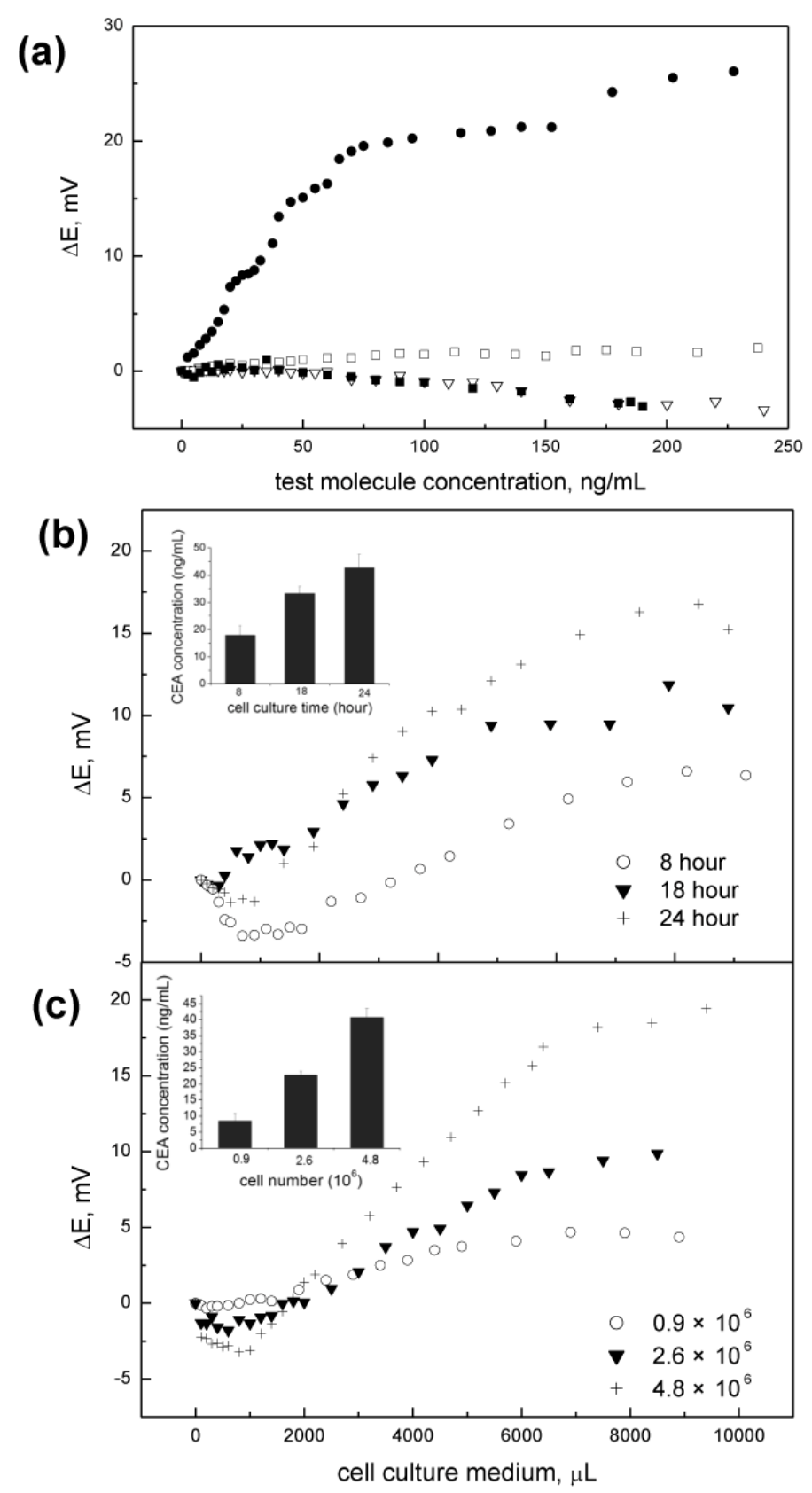

Fig. 3. Potentiometric measurement of carcinoembryonic antigen (CEA).

We used a CEA-specific sensing element to determine the levels of CEA as in Methods ${ }^{*}$. (a) Sensor response as a function of the concentration of the target molecules: specific CEA sensor response to purified CEA $(\bullet)$ or hemoglobin $(\nabla)$; control

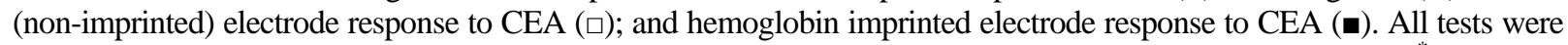
performed in Ham's F-12K medium supplemented with $10 \%$ fetal bovine serum as described in Methods*. (b) CEA sensor response in culture medium as a function of the added medium volume; cells were cultured for different periods of time. Inset: CEA concentration in cell culture medium determined by immunoassay. The cell number per plate: $3 \times 10^{6}$ to $4 \times 10^{6}$. (c) CEA sensor response in cell culture medium as a function of the added medium volume. The number of cells differed among plates. Inset: CEA concentration in cell culture medium determined by immunoassay. Cell culture time: 20-24 h.

*Methods are described in the following reference from which the figure is reproduced. (Reprinted with permission from Wang, Y. T., Zhang, Z. Q., Jain, V., Yi, J. J., Mueller, S., Sokolov, J., Liu, Z. X., Levon, K., Rigas, B. and Rafailovich, M. H. 2010, Sensors and Actuators B-Chemical, 146(1), 381-387). 


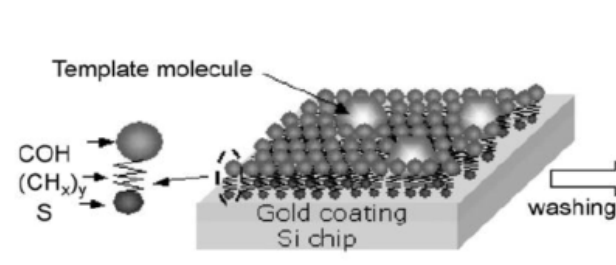

(a)

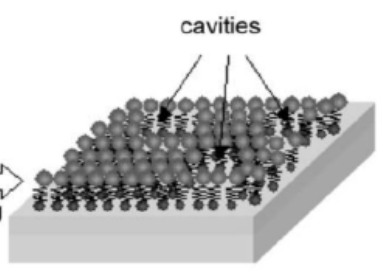

(b)

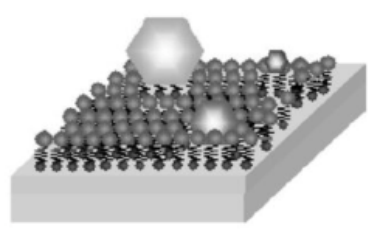

(c)

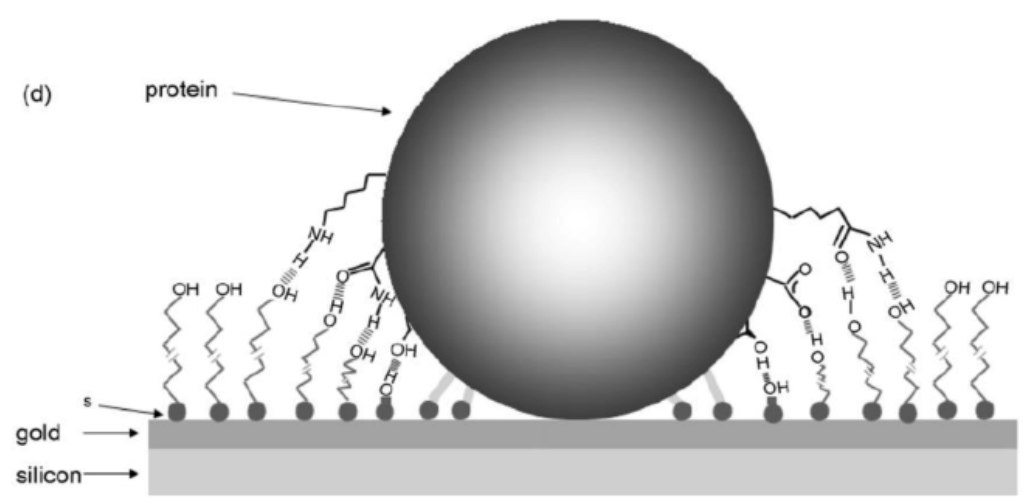

Fig. 4. Fabrication of protein-imprinted SAM sensor and its proposed working mechanism.

(a) Co-adsorption of template protein molecules and thiol SAMs onto the gold surface.

(b) Cavities created after washing off the templates.

(c) Selective adsorption of the template protein molecules against other molecules.

(d) Hypothetical binding mechanism showing the hydrogen bonds between protein and -OH end groups of the thiol.

(Reprinted with permission from Wang, Y. T., Zhou, Y. X., Sokolov, J., Rigas, B., Levon, K. and Rafailovich, M. 2008, Biosensors \& Bioelectronics, 24(1), 162-166).

alkanethiol SAM on the gold surface makes the hydrogen bonding to the appropriate groups of amino acid from hydrophilic regions of the target protein possible and, in addition, this part of interaction helps retaining the conformation of the protein instead of denaturing it by the strong attraction force exerted by the substrate; at the same time, the amino acid from hydrophobic regions are adsorbed to the gold surface through hydrophobic interactions and/or electrostatic forces [91]. These forces are sufficient to attract the target molecules from the solution, but weaker than covalent bonds and hence allow their removal following the templating process [93]. The cavities produced by removing the template maintain the shape of the molecule of the surface amino acid groups. Since this shape is specific and local, recognition of the analyte can be achieved even if only a small fraction of the protein surface presents at the templated electrodes [94]. For other guest molecules without these complementarities, two possibilities exist: (1) for the smaller molecules, non-reversible adsorption onto the gold substrate happens, which is highly likely to denature the proteins and change their charges [95]; (2) for the larger molecules, they do not fit into the cavities and no adsorption occurs due to the resistance of the hydrophilic SAM. The series of studies have not only revealed the possibility and potential of the SMI technique in selectively adsorbing bio-macromolecules in spite of the fact that the size of the template molecules is much larger than the SAM thickness, but more importantly, they have extended the concept of the molecular imprinting from the exact match in size, shape and functional groups to a re-adsorption mechanism of a partial match and memory effect by thehydrophobic/hydrophilic interaction, and hence greatly advanced the potential of the technique in terms of diversity of detecting species, simplification of fabrication conditions, as well as ease of application. Despite the effectiveness of the technique in these bio-macromolecules, the specific mechanism is at the hypothesis stage, and in-depth study to characterize the adsorption and 
removal of the protein/viruses is needed to prove the proposed mechanism, and has been the focus of the group in subsequent investigation.

Mathur et al. used the surface imprinting to build a biosensor for the detection of the pleural mesothelioma cancer marker hyaluronan-linked protein 1 (HAPLN1) [58]. In their study, they first used the bovine serum albumin (BSA) as a model protein to find out the optimal conditions required to imprint the similar molecular weight protein HAPLN1. Then, matrix-assisted laser desorption ionization-time of flight mass spectrometry (MALDI-TOF MS) was used to confirm selectivity of imprints. Molecular imprinting-based biosensor was successfully built to recognize HAPLN1 and it demonstrated high sensitivity by detecting low concentration of biomarker in serum/buffer solution. The sensor showed a limit of detection of picomolar concentrations with a response time of 2-5 minutes.

\section{Discussion of the role of surface affinity, roughness, and chemistry}

After Wang et al., a further study on a SMI sensor used for CEA detection and the exploration of imprinting mechanism was carried out by $\mathrm{Yu}$ et al. in the same group. The technique was expanded to detect CEA in pancreatic cyst fluid from patients, giving the technique more clinical relevance [14]. A revised quasi-3D molecular imprinting mechanism was proposed in which the roughness of the gold substrate gives additional dimension to the complementary of the template molecules to the surface and SAM matrix (Fig. 5). Since the goldcoated silicon substrate is not perfectly smooth, but has a surface roughness of approximately RMS 3-4 nm, when template bio-macromolecules and the thiol molecules co-adsorbed onto the surfaces, the quasi-3D dimensional surface provides shape specificity of the template molecules in addition to hydrophobic/hydrophilic interactions of different areas; the removal of the template molecules will

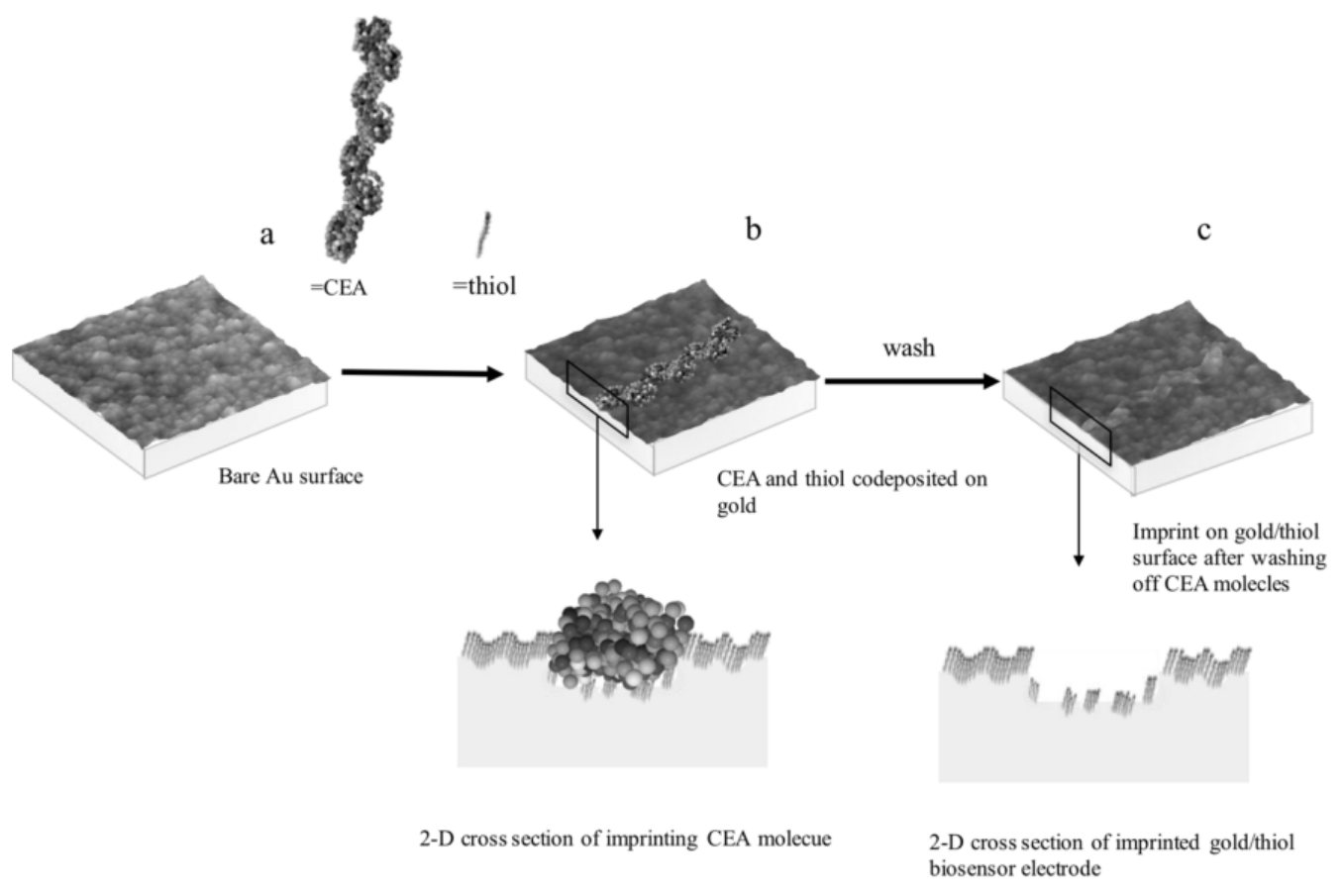

Fig. 5. Updated CEA 3D molecular imprinting model.

(a) Co-adsorption of template protein molecules and thiol SAMs onto the gold surface.

(b) Cavities created after washing off the templates.

(c) Selective adsorption of the template protein molecules against other molecules.

(Reproduced from Yu, Y. J., Zhang, Q., Buscaglia, J., Chang, C. C., Liu, Y., Yang, Z. H., Guo, Y. C., Wang, Y. T., Levon, K. and Rafailovich, M. 2016, Analyst, 141, 4424 with permission from The Royal Society of Chemistry). 
leave behind the crevices that the target molecules with the same shape and conformation have a higher tendency to re-fit into. Compared to the previously proposed 2D imprinting model, the quasi-3D imprinting model could better explain why a SAM with approximately only $1 \mathrm{~nm}$ thickness could imprint larger macromolecules with a size of 3-5 nm. Natural surface roughness allows the macromolecules to be enveloped by the templating SAM. This co-deposition process was characterized by cyclic voltammetry (CV) and electrochemical impedance spectra (EIS) (Fig. 6). In the $\mathrm{CV}$ test, redox peaks of $\left[\mathrm{Fe}(\mathrm{CN})_{6}\right]^{4-}$ and $\left[\mathrm{Fe}(\mathrm{CN})_{6}\right]^{3-}$ from electron transfer between gold electrode and electroactive moieties in solution were tracked, and observed to significantly decrease with the adsorption of the thiol and the protein, indicating the formation of condensed non-conductive thiol/CEA layer that hindered the electron transfer process; after removal of the CEA by selective washing, the peaks resumed, suggesting a high percentage of the CEA molecules were removed from the electrode surface; finally, upon the addition of $8.5 \mathrm{ng} \mathrm{mL}^{-1}$ of into the solution, the peaks were reduced again, which suggested an efficient recognition of CEA by a large faction of the cavities and a partial occupation of those by the CEA molecules. The EIS experiments measuring the electron transfer resistance $\left(\mathrm{R}_{\mathrm{et}}\right)$ of the gold electrode reached the same conclusion as well. The potentiometric response of the sensor $(\Delta)$ was also studied as a function of the concentration of the CEA in the imprinting solution (c), and was found to increase with $c$ till $15 \mathrm{ng} / \mathrm{mL}$, below which both the sensitivity and maximum response increases with $c$, above which the response no longer increases. That suggested the optimal template concentration at which the largest number of cavities were created on the sensor surface. To further validate this molecular imprinting mechanism, thiol titration experiments were designed based on the electron transfer reaction. Robust potentiometric signal can be obtained for the cavities formed at $15 \mathrm{ng} \mathrm{ml}^{-1}$, which is consistent with the CEA optimal imprinting condition as well. ELISA was also applied to estimate the mass of CEA absorption on gold surface, showing the similar absorption efficiency in DI water as well as MI solution. After careful calibration with ELISA test, a standard curve for CEA can be obtained, allowing the application of CEA biosensor to complicated physiological environment. The patient cyst fluid
(A)

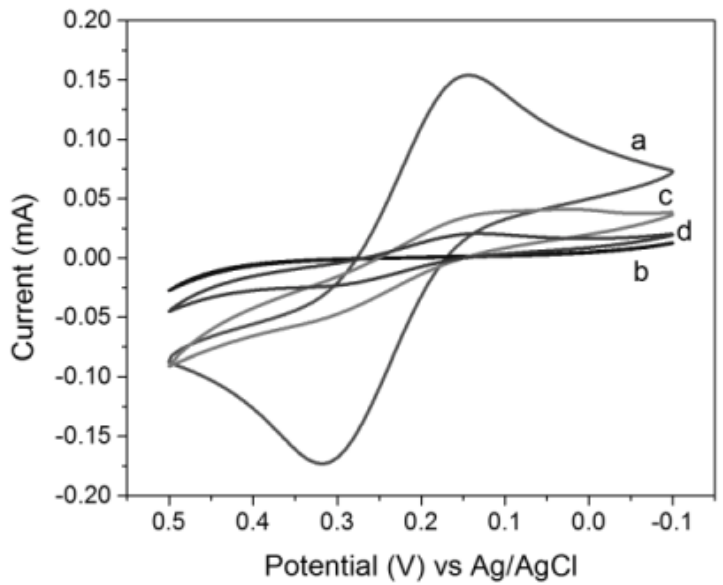

(B)

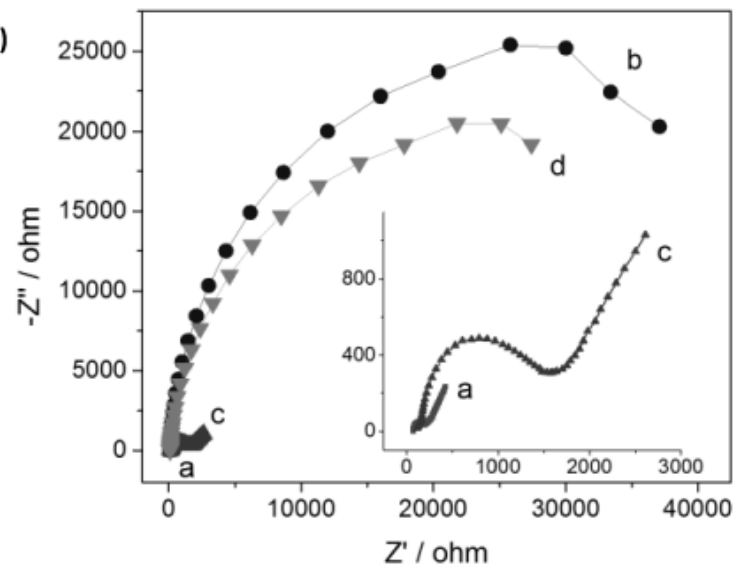

Fig. 6. (A) Cyclic voltammograms (CV) of the different electrodes: (a) bare gold, (b) after CEA imprint, (c) after washing, (d) after addition of CEA (8 ng/ml); Scan rate, $0.1 \mathrm{~V} / \mathrm{s}$. (B) Electrochemical impedance spectra (EIS) of different electrodes: (a) bare gold, (b) after CEA imprint, (c) after washing, (d) after addition of CEA (8 ng/ml). Inset: EIS of (a) bare gold (c) and after washing. EIS was tested with $5 \mathrm{mV}$ amplitude and frequency range of 0.1-100,000 Hz. Both CV and EIS were performed in $\mathrm{KCl}$ solution $(0.1 \mathrm{M})$ containing $\mathrm{Fe}(\mathrm{CN})_{6}^{3-/ 4-}(5 \mathrm{mM})$. (Reproduced from Yu, Y. J., Zhang, Q., Buscaglia, J., Chang, C. C., Liu, Y., Yang, Z. H., Guo, Y. C., Wang, Y. T., Levon, K. and Rafailovich, M. 2016, Analyst, 141, 4424 with permission from The Royal Society of Chemistry).

sensing data obtained using molecular imprinting biosensor fits well with that from the ELISA method, demonstrating the potential of this method in clinical diagnosis.

In order to further apply the SMI biosensor to a broad spectrum of biomolecules, surface roughness, as a contributing factor for SMI biosensor, 
needs to be thoroughly investigated. Therefore, $\mathrm{Yu}$ et al. continued to propose that both the sensitivity and selectivity could be optimized as long as the surface roughness was tailored to match the size of the analyte. Multi-scale roughness gold surfaces with a large horizontal and vertical size distribution as achieved by physical vapor deposition (PVD) on smooth and rough silicon substrates, have been applied in the potentiometric detection of a large size range of analytes, ranging from protein of a few nanometers to bacteria of hundreds of nanometers. To systematically study the analytes and the corresponding roughness surfaces, more than ten types of analytes were used.

In Fig. 7(A), the relative size of the analytes is compared and plotted, ranging from $5 \mathrm{~nm}$ to $2 \mu \mathrm{m}$. As long as the size of the analyte matches with the surface roughness, excellent sensitivity and specificity of the biosensor could be achieved.
On the top of Fig. 7(A), optimized surface roughness is indicated for each analyte. The corresponding fitting model on both surface for small and large molecules are illustrated in Fig. 7(B). It is clear that small molecule can fit well on smooth gold surface, whereas for the large molecule, it fits well only on rough surfaces. Once the analyte fits well to the surface roughness, larger potentiometric signal can be achieved. However, poor response can occur when the size of the analyte mismatches with the size of niche on the surface. This scheme vividly shows how surface roughness could significantly influence the quality of the imprinting, indicating the importance of matching the surface roughness with the size of analytes.

In conclusion, all of these studies prove the importance of understanding how the surface roughness governs the efficiency of molecular imprinting process. Although the actual molecular

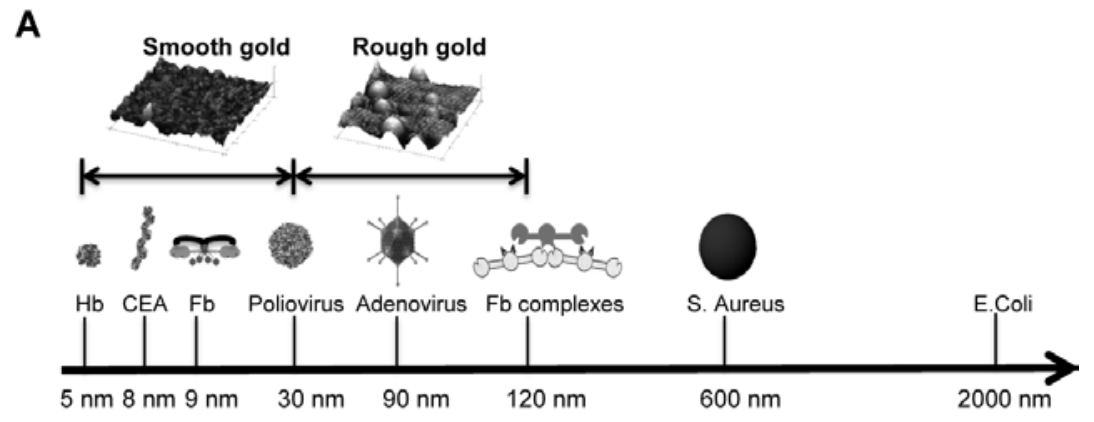

B

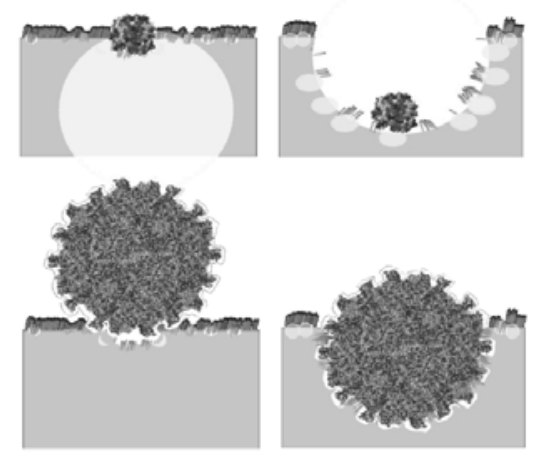

Fig. 7. (A) Size distribution covering all the analytes in this study, including protein, antigen, virus and bacteria, as plotted on a nanometer scale chart. (B) Schematic representation of the fitting principle of small and large analytes into imprints on multi-scale roughness surface. A good fit occurs only when the analyte dimension matches with the gold surface roughness. (Reproduced from Yu, Y. J., Zhang, Q., Chang, C. C., Liu, Y., Yang, Z. H., Guo, Y. C., Wang, Y. T., Galanakis, D. K., Levon, K. and Rafailovich, M. 2016, Analyst, 141, $5607-5617$ with permission from The Royal Society of Chemistry). 
imprinting surface is still hard to visualize and characterize, the result here shows how quasi-3D model could influence the detection. More direct surface measurements are required to be applied here to better investigate this aspect of the molecular imprinting process.

\section{Conclusions and future outlook}

SMI using the SAMs on gold substrates has proven successful in building sensors for the detection of several bio-macromolecules including globular proteins, cancer biomarkers and viruses. The fabrication conditions such as the solvent types and blend ratios of aqueous/organic parts, the concentration of the organic molecules and the template bio-macromolecules, the washing method, immersion time, and the $\mathrm{pH}$ of the solution, as well as the surface roughness of the gold substrate, are all crucial in determining the function and the sensitivity of the sensor. The proposed mechanism has been verified by various methods: AFM, contact angle goniometry and FT-IR have been used to confirm the formation, crystallinity and stability of thiol SAMs; cyclic voltammetry (CV), electrochemical impedance spectra (EIS), and MALDI-TOF MS have been used to monitor the adsorption, removal and re-adsorption of the template bio-macromolecules. The quantification capability of the SMI sensor for CEA has been verified by comparing with ELISA in a clinical study of pancreatic cyst fluid. The high sensitivity, good selectivity and rapid response have made SMI a promising technique for the real-time detection of biomarkers, biological war-fare agents, as well as fast screening of diseases. Application of the technique in real medical diagnosis calls for improvements in several aspects including user-friendly sampling technique for in-situ detection, advanced algorithm for result screening, improved stability and re-usability, as well as proven viability in more biomolecules species, which are all under active study to turn SMI into a widely applied, robust and reliable sensor technology in the foreseeable future.

\section{ACKNOWLEDGEMENTS}

Support by the National Science Foundation INSPIRE program NSF-1344267 and the NY Center for Advanced Technology in Sensors is gratefully acknowledged.

\section{CONFLICT OF INTEREST STATEMENT}

There are no conflicts of interest.

\section{REFERENCES}

1. Sai, V. V. R., Mahajan, S., Contractor, A. Q. and Mukherji, S. 2006, Analytical Chemistry, 78, 8368.

2. Rich, R. L. and Myszka, D. G. 2006, Journal of Molecular Recognition, 19, 478.

3. Aray, A., Chiavaioli, F., Arjmand, M., Trono, C., Tombelli, S., Giannetti, A., Cennamo, N., Soltanolkotabi, M., Zeni, L. and Baldini, F. 2016, Journal of Biophotonics, 9, 1077.

4. Poturnayova, A., Castillo, G., Subjakova, V., Tatarko, M., Snejdarkova, M. and Hianik, T. 2017, Sensors and Actuators B-Chemical, 238, 817.

5. Gianneli, M., Tsougeni, K., Grammoustianou, A., Tserepi, A., Gogolides, E. and Gizeli, E. 2016, Sensors and Actuators B-Chemical, 236, 583.

6. Ricco, A. J., Crooks, R. M. and Osbourn, G. C., 1998, Accounts of Chemical Research, 31, 289.

7. Yu, Y., Wu, T., Johnson-Buck, A., Li, L. and Su, X. 2016, Biosensors and Bioelectronics, 82, 248.

8. Kabashima, T., Yu, Z., Tang, C., Nakagawa, Y., Okumura, K., Shibata, T., Lu, J. and Kai, M., 2008, Peptides, 29, 356.

9. Chen, J., Jackson, A. A., Rotello, V. M. and Nugen, S. R. 2016, Small, 12, 2469.

10. Wang, Y. T., Zhang, Z. Q., Jain, V., Yi, J. J., Mueller, S., Sokolov, J., Liu, Z. X., Levon, K., Rigas, B. and Rafailovich, M. H. 2010, Sensor Actuat B-Chem, 146, 381.

11. Moghadam, M. R., Akbarzadeh, S. and Nasirizadeh, N. 2016, Microchimica Acta, 183, 1069.

12. Busono, P. 2015, In: $7^{\text {th }}$ Wacbe World Congress on Bioengineering, J. Goh and C. T. Lim (Eds.), 52, 134.

13. Yu, Y. J., Zhang, Q., Chang, C. C., Liu, Y., Yang, Z. H., Guo, Y. C., Wang, Y. T., Galanakis, D. K., Levon, K. and Rafailovich, M. 2016, Analyst, 141, 5607.

14. Yu, Y. J., Zhang, Q., Buscaglia, J., Chang, C. C., Liu, Y., Yang, Z. H., Guo, Y. C., Wang, Y. T., Levon, K. and Rafailovich, M. 2016, Analyst, 141, 4424. 
15. Wang, Y. T., Zhou, Y. X., Sokolov, J., Rigas, B., Levon, K. and Rafailovich, M. 2008, Biosensors and Bioelectronics, 24, 162.

16. Zhang, Q., Majumdar, H. S., Kaisti, M., Prabhu, A., Ivaska, A., Osterbacka, R., Rahman, A. and Levon, K. 2015, Ieee T Electron Dev, 62, 1291.

17. Miao, P., Wan, B., Yu, Z., Zhao, J. and Tang, Y. 2015, Biosensors and Bioelectronics, 63, 365.

18. Zhao, P., Zhou, L., Nie, Z., Xu, X., Li, W., Huang, Y., He, K. and Yao, S. 2013, Anal. Chem., 85, 6279.

19. Chen, J., Jiang, Z., Ackerman, J. D., Yazdani, M., Hou, S., Nugen, S. R. and Rotello, V. M. 2015, Analyst, 140, 4991.

20. Chen, J. H., Zhou, Y. L., Wang, D. H., He, F., Rotello, V. M., Carter, K. R., Watkins, J. J. and Nugen, S. R. 2015, Lab Chip, 15, 3086.

21. Wang, D. H., Wang, Z. Y., Chen, J. H., Kinchla, A. J. and Nugen, S. R. 2016, Food Control, 62, 81.

22. Dai, M., Chen, J., Goddard, J. M. and Nugen, S. R. 2017, Nanotechnology, 28, 06LT01.

23. Kaisti, M., Zhang, Q. and Levon, K. 2017, Sensors and Actuators B-Chemical, 241, 321.

24. van Grinsven, B., Betlem, K., Cleij, T. J., Banks, C. E. and Peeters, M. 2017, Journal of Molecular Recognition, 30, e2563.

25. Akyilmaz, E., Erdogan, A., Ozturk, R. and Yasa, I. 2007, Biosensors and Bioelectronics, 22, 1055.

26. Pan, L. H., Kuo, S. H., Lin, T. Y., Lin, C. W., Fang, P. Y. and Yang, H. W. 2017, Biosensors and Bioelectronics, 89, 598.

27. Jabbari, S., Dabirmanesh, B., Arab, S. S., Amanlou, M., Daneshjou, S., Gholami, S. and Khajeh, K. 2017, Sensors and Actuators B-Chemical, 240, 519.

28. Janegitz, B. C., Silva, T. A., Wong, A., Ribovski, L., Vicentini, F. C., Sotomayor, M. D. T. and Fatibello, O. 2017, Biosensors and Bioelectronics, 89, 224.

29. Zhang, W. and Serpe, M. J. 2017, Sensors and Actuators B-Chemical, 238, 441.

30. Zhang, Q., Prabhu, A., San, A., Al-Sharab, J. F. and Levon, K. 2015, Biosensors and Bioelectronics, 72, 100.

31. Wei, X., Panindre, P., Zhang, Q. and Song, Y. A. 2016, Acs Sensors, 1, 862.
32. Li, Z. Q., Liu, C. C., Zhang, D. W., Luo, S. P. and Yamaguchi, Y. 2016, J Chromatogr. B, 1011, 114.

33. Mukherjee, K., Bhattacharyya, S. and Peralta-Yahya, P. 2015, Acs Synthetic Biology, 4, 1261.

34. Holowko, M. B., Wang, H. J., Jayaraman, P. and Poh, C. L. 2016, Acs Synthetic Biology, 5, 1275.

35. Li, Z. Q., Li, D., Zhang, D. W. and Yamaguchi, Y. 2014, Analyst, 139, 6113.

36. Li, Z. Q., Yang, R. L., Wang, Q., Zhang, D. W., Zhuang, S. L. and Yamaguchi, Y. 2015, Anal. Biochem., 471, 70.

37. Yu, Z., Kabashima, T., Tang, C., Shibata, T., Kitazato, K., Kobayashi, N., Lee, M. K. and Kai, M. 2010, Anal. Biochem., 397, 197.

38. Lines, J. A., Yu, Z. Q., Dedkova, L. M. and Chen, S. X. 2014, Biochem. Bioph. Res. Co, 443, 308.

39. Chen, J. H., Alcaine, S. D., Jiang, Z. W., Rotello, V. M. and Nugen, S. R. 2015, Analytical Chemistry, 87, 8977.

40. Wang, Z. Y., Wang, D. H., Chen, J. H., Sela, D. A. and Nugen, S. R. 2016, Analyst, 141, 1009.

41. Chen, J., Andler, S. M., Goddard, J. M., Nugen, S. R. and Rotello, V. M. 2017, Chem. Soc. Rev., 46, 1272.

42. Li, Z. Q., Zhao, Y., Zhang, D. W., Zhuang, S. L. and Yamaguchi, Y. 2016, Sensors and Actuators B-Chemical, 230, 779.

43. Li, Z. Q., Liu, C. C., Ma, S. Y., Zhang, D. W. and Yamaguchi, Y. 2016, Anal MethodsUK, 8, 2330.

44. Kumar, T. A., Capua, E., Tkachev, M., Adler, S. N. and Naaman, R. 2014, Adv. Funct. Mater., 24, 5833.

45. Guo, L. H., Zhou, X. D. and Kim, D. H. 2011, Biosensors and Bioelectronics, 26, 2246.

46. Goertz, M. P., Goyal, N., Montano, G. A. and Bunker, B. C. 2011, Langmuir, 27, 5481.

47. He, C. F., Wang, S. H., Yu, Y. J., Shen, H. Y., Zhao, Y., Gao, H. L., Wang, H., Li, L. L. and Liu, H. Y. 2016, Cancer Biol. Med., 13, 299.

48. Owens, P. K., Karlsson, L., Lutz, E. S. M. and Andersson, L. I. 1999, Trac-Trend Anal. Chem., 18, 146. 
49. Lieberzeit, P. A. and Dickert, F. L., 2008, Anal. Bioanal. Chem., 391, 1629.

50. Haupt, K., Dzgoev, A. and Mosbach, K. 1998, Analytical Chemistry, 70, 628.

51. Mosbach, K. 2006, Scientific American, 295, 86.

52. Dickert, F. L., Lieberzeit, P. and Tortschanoff, M. 2000, Sensors and Actuators B-Chemical, 65, 186.

53. Ekberg, B. and Mosbach, K. 1989, Trends Biotechnol, 7, 92.

54. Wulff, G. 1995, Angewandte ChemieInternational Edition in English, 34, 1812.

55. Turner, N. W., Jeans, C. W., Brain, K. R., Allender, C. J., Hlady, V. and Britt, D. W. 2006, Biotechnol. Prog., 22, 1474.

56. Ye, L. and Mosbach, K. 2008, Chem. Mater., 20, 859.

57. Zhang, X., Du, X., Huang, X. and Lv, Z. 2013, J. Am. Chem. Soc., 135, 9248.

58. Mathur, A., Blais, S., Goparaju, C. M. V., Neubert, T., Pass, H. and Levon, K. 2013, Plos One, 8, e57681.

59. Sellergren, B., 2000, Angew. Chem. Int. Ed. Engl., 39, 1031.

60. Matsui, J., Akamatsu, K., Hara, N., Miyoshi, D., Nawafune, H., Tamaki, K. and Sugimoto, N. 2005, Analytical Chemistry, 77, 4282.

61. Wang, J. L. and Zhou, H. S. 2008, Analytical Chemistry, 80, 7174.

62. Miao, P., Tang, Y. and Yin, J. 2015, Chem Commun (Camb), 51, 15629.

63. Gorton, L. 2005, Biosensors and Modern Biospecific Analytical Techniques, Chapter 1 by Schlereth, D. D., Elsevier.

64. Ulman, A. 1996, Chemical Reviews, 96, 1533.

65. Bain, C. D. and Whitesides, G. M., 1989, Journal of the American Chemical Society, 111, 7164.

66. Sagiv, J. 1980, Journal of the American Chemical Society, 102, 92.

67. Li, S. S., Yang, D. Y., Tu, H. Y., Deng, H. T., Du, D. and Zhang, A. D. 2013, Journal of Colloid and Interface Science, 402, 284.

68. Takehara, K., Ide, Y., Aihara, M. and Obuchi, E., 1992, Bioelectrochemistry and Bioenergetics, 29, 103.

69. Zhang, L. T., Lu, T. B., Gokel, G. W. and Kaifer, A. E. 1993, Langmuir, 9, 786.

70. Dubois, L. H. and Nuzzo, R. G. 1992, Annual Review of Physical Chemistry, 43, 437.
71. Troughton, E. B., Bain, C. D., Whitesides, G. M., Nuzzo, R. G., Allara, D. L. and Porter, M. D. 1988, Langmuir, 4, 365.

72. Finklea, H. O., Snider, D. A., Fedyk, J., Sabatani, E., Gafni, Y. and Rubinstein, I. 1993, Langmuir, 9, 3660.

73. Hickman, J. J., Ofer, D., Zou, C. F., Wrighton, M. S., Laibinis, P. E. and Whitesides, G. M. 1991, Journal of the American Chemical Society, 113, 1128.

74. Schlenoff, J. B., Li, M. and Ly, H. 1995, Journal of the American Chemical Society, $117,12528$.

75. Linford, M. R. and Chidsey, C. E. D. 1993, Journal of the American Chemical Society, $115,12631$.

76. Cao, G., Hong, H. G. and Mallouk, T. E. 1992, Accounts of Chemical Research, 25, 420.

77. Flink, S., van Veggel, F. and Reinhoudt, D. N. 2000, Advanced Materials, 12, 1315.

78. Ricco, A. J. and Crooks, R. M. 1998, Accounts of Chemical Research, 31, 200.

79. Netzer, L. and Sagiv, J. 1983, Journal of the American Chemical Society, 105, 674.

80. Netzer, L., Iscovici, R. and Sagiv, J. 1983, Thin Solid Films, 99, 235.

81. Netzer, L., Iscovici, R. and Sagiv, J. 1983, Thin Solid Films, 100, 67.

82. Cohen, S. R., Naaman, R. and Sagiv, J. 1986, Journal of Physical Chemistry, 90, 3054.

83. Sagiv, J. 1979, Israel Journal of Chemistry, 18, 339.

84. Sagiv, J. 1979, Israel Journal of Chemistry, 18, 346.

85. Zhou, Y. X., Yu, B. and Levon, K. 2005, Biosensors and Bioelectronics, 20, 1851.

86. Zhou, Y. X., Yu, B., Levon, K. and Nagaoka, T. 2004, Electroanalysis, 16, 955.

87. Zhou, Y. X., Yu, B., Shiu, E. and Levon, K., 2004, Analytical Chemistry, 76, 2689.

88. Duffy, M. J. 2001, Clinical Chemistry, 47, 624.

89. Mosser, A. G., Sgro, J. Y. and Rueckert, R. R. 1995, Acta Crystallographica Section DBiological Crystallography, 51, 490.

90. Ostuni, E., Chapman, R. G., Holmlin, R. E., Takayama, S. and Whitesides, G. M. 2001, Langmuir, 17, 5605. 
91. Kaufman, E. D., Belyea, J., Johnson, M. C., Nicholson, Z. M., Ricks, J. L., Shah, P. K., Bayless, M., Pettersson, T., Feldoto, Z., Blomberg, E., Claesson, P. and Franzen, S. 2007, Langmuir, 23, 6053.

92. Tang, Q., Xu, C. H., Shi, S. Q. and Zhou, L. M. 2004, Synthetic Metals, 147, 247.
93. Paleos, C. M. and Tsiourvas, D. 1997, Advanced Materials, 9, 695.

94. Shi, H. Q., Tsai, W. B., Garrison, M. D., Ferrari, S. and Ratner, B. D. 1999, Nature, 398, 593.

95. Chen, X. X., Ferrigno, R., Yang, J. and Whitesides, G. A. 2002, Langmuir, 18, 7009. 